\title{
Outpatient (Ambulatory) Pharmacy; an Innovation in Dispensing System to Optimize Performance and Meet Standards
}

\section{Emad I. Hammouda ${ }^{1 *}$ and Sarah E. Hammouda ${ }^{2}$}

${ }^{1}$ Tawam Hospital/ Johns Hopkins Medicine International, Al Ain City, UAE

${ }^{2}$ Pharmacy college, Al Ain University, UAE

\author{
Abstract \\ to hospital customers: \\ premises \\ 2. Dispensing of home care medications \\ 3. Generation of invoices for payment according to agreed payment systems \\ 4. Medication reconciliation \\ 5. Patient and family Education \\ 6. Counseling patients on related medication regimens \\ 7. Offering retail sale of health-related materials \\ 8. Handling and managing patients excess or old medications \\ 9. Home medication review \\ 10. Documentation of daily pharmacy practice
}

Services and dynamic activities of hospital pharmacy are affecting public health and welfare. Outpatient pharmacy is a section of hospital pharmacy department that is required to provide the following scope of services and activities

1. Delivery of medication-related services to outpatients and health care providers working within hospital

Quality of pharmacy services is surely influenced by organization priorities, targets and leadership commitments [1]. Relentless efforts have been made through last decades to gain patients and healthcare organizations satisfaction regarding patients waiting time, effective counseling and reduction of medication errors without a noticeable success. A breakthrough innovation in dispensing system is needed to overcome this nagging issue in a reliable and comprehensive fashion. The above mentioned services are regarded as a prerequisite to improve quality, work flow and promote customer's satisfaction and this can be achieved through three basic elements including:

1. Adaptive design

2. Qualified and well trained staff

3. An effective process

The mission is to provide a pharmacy service that is able to achieve "improved health and well-being, best quality of care and value for all stakeholders". The innovation in dispensing system is optimistically targeted to achieve this mission and keeps extended open venues for improvement in pharmacy performance.

Keywords: Outpatient (ambulatory) pharmacy; Waiting time; Counseling; Reconciliation; Reengineering; safety; The 8 steps system; Customer satisfaction; Design

\section{Outpatient Pharmacy Scope of Services}

Hospital ambulatory (outpatient) pharmacy has a set of services provided to ambulatory patients, home care patients, hospital staff and probably emergency department patients depending on the level of care of an organization. Ambulatory refers to patients not occupying beds in hospitals or other inpatient settings. Pharmacy department's vision is to be a reliably standard setting in its provision of direct patient care and to continuously develop methods that improve this practice. Pharmacy department facilitates safe, effective, efficient and economical use of medications with the aim of optimizing patient care by qualified and trained staff. Pharmacists play an essential role in the safe, quality and effective use of medications in improving patient's physical and mental wellness. They are instrumental in managing medication-related issues to complement the holistic care for patients throughout the organization. Pharmacists provide education to patients and caregivers on the safe and appropriate use of medications, counsel on medication compliance, monitor and manage medication side effects, as well as screen for dangerous drug interactions. In addition, they specialize in recommending optimal medication therapy for concurrent medical problems, with the aim of enhancing treatment outcomes and facilitating the continuity of care as patients integrate back into the community. Outpatient pharmacy basic services and functions may include:

\section{Dispensing}

That is the delivery of product-related and clinical services to patients and health care providers. Dispensing is the procedures required in completing the filling of prescriptions (e.g., checking, pouring, counting, packaging, labeling, review....etc.). The fundamental concept is to give the right medication to the right

Corresponding author: Emad I. Hammouda, Pharmacy Manager in Wagan Hospital / a division of Tawam Hospital/ Johns Hopkins Medicine International, A Ain City, Po Box: 15258, UAE, Tel: + 971037017 852; Fax: + 971037017 732 Email:emhammouda@hotmail.com

Received November 16, 2011; Accepted March 30, 2012; Published April 02 2012

Citation: Hammouda EI, Hammouda SE (2012) Outpatient (Ambulatory) Pharmacy; an Innovation in Dispensing System to Optimize Performance and Meet Standards. Pharmaceut Reg Affairs 1:101. doi:10.4172/2167-7689.1000101

Copyright: (c) 2012 Hammouda El, et al. This is an open-access article distributed under the terms of the Creative Commons Attribution License, which permits unrestricted use, distribution, and reproduction in any medium, provided the original author and source are credited. 
patient after proper identification. Drugs should be dispensed at the right dose, route of administration, form and duration of treatment. Evaluation of medication use and dosing on an individual basis for each patient, consultation with the physician regarding appropriate plan of care regarding drug use, discharge planning, implementing and helping to evaluate cost containment or drug therapy initiatives and the management of drug formulary of the P\&T Committee are all ongoing practice with prescription dispensing. Pharmacy staff works in close collaboration with the different medical departments, nursing services and dietary department to meet customer needs. Today, most leading healthcare institutions are trying to adopt patient-focused clinical pharmacy practice by assigning clinical pharmacists to patient care and giving them an active role in the healthcare team. Ambulatory pharmacy, as a dynamic healthcare unit has a great potential to promote rational drug use for patients without over or underuse of drugs. Ideally, outpatient pharmacy should be opened 24/7, with professional staff always on hand to assist customers. Constantly, pharmacy management should upgrade and extend services to ensure that all patients receive the best pharmaceutical care possible.

\section{Delivery of home care medications}

Usually home care prescriptions are dispensed and refilled from the ambulatory pharmacy as a service to this patient population. Assessment of drug therapy is done during each dispensing of a new or refilled prescription including allergies, adherence, duplicate therapy, untreated conditions and over/under-dosing, taking renal and hepatic function into account. Drug-drug, drug-food, drug-disease interactions are checked as well.

\section{Billing}

Pharmacy staff generates invoices for payment or co- payment of prescribed drugs according to an agreed system and contracts between involved parties.

\section{Medication reconciliation}

Medication reconciliation is the process of comparing a patient's new medication orders to all of the medications and complementary products that the patient has been taking. This reconciliation is done to avoid medication errors such as omissions, duplications, dosing errors, or drug interactions. It should be done at every transition of care in which new medications are ordered or existing orders are rewritten. Transitions in care include changes in setting, service, practitioner or level of care. This process comprises five steps:

1) Developing a list of current medications.

2) Developing a list of medications to be prescribed.

3) Comparing the medications on the two lists.

4) Making clinical decisions based on the comparison.

5) Communicating the new list to appropriate caregivers and to the patient and family after each visit or discharge (Form A).

Accurate, complete and ongoing medication reconciliation can prevent numerous potential prescribing and administration errors. Failure to reconcile medications may occur by the practice of writing orders, such as "resume pre-op medications," which are highly error prone and are known to result in adverse drug events. Medication errors related to medication reconciliation typically occur at the interfaces of care when a patient is admitted, transferred, or discharged from a health care facility.

\section{Patient and family education}

Pharmacists are qualified to answers questions to customers about prescriptions and medications. Care to the outpatient arena should be provided with the emphasis on continuity of care. A continuum of clinical service settings has to be established so that patient care can be provided in the most cost-effective setting that is clinically appropriate. There is also a need to tailor medication treatment solutions to patient oriented local circumstances. Pharmacists have to be precise in their operations and require maximum use of allocated space to allow for efficient functioning and be able to perform the expected high level of service to patients and their families. Outpatient capacity should be set in a way to accommodate the workload shifted from inpatient to an outpatient setting and to obviate the need for inpatient care as much as possible. Education and referral for patients included or excluded from clinical trials should be maintained during all patients encounters in outpatient pharmacy.

\section{Patient counseling}

Individual and group counseling for patients living with chronic or special care illnesses or newly diagnosed and concerned about coping with their own or partner illnesses should receive proper focused counseling. Partner notification, family support, use of community resources, and long-term psychosocial support are options to optimize pharmacotherapy in outpatient pharmacy. Provision of patientcentered, cost-effective care through a pharmacy designed care plan should be continually done to pharmacy clients by excellence in pharmaceutical care at a level that meets the needs and expectations of patients in such a manner as to promote their health and well-being and to ensure that the performance of duties and responsibilities involved in the drug use process are in accordance with relevant laws, rules, regulations, and accreditation standards.

\section{Retail sale}

Outpatient pharmacy offers a retail sale of consumables, complementary products and health-related materials e.g. movement aids, relaxation manuals, health guide books etc.

\section{Managing patients excess or old medications}

Sometimes called "yellow bag service". This service is meant for patients who have excess or old medications that they are unsure about the safe and/or the appropriate use. Pharmacy staff provides medication sorting service as well as information on medication proper storage and use. This service is offered at both outpatient settings to interested customers.

\section{Home medication review}

This is a domiciliary medication management review offered to selected identified patient population. It is run as an agreed treatment plan in coordination with home care team. A Home medicines review is a procedure in which an assigned pharmacist (a member of the home care team) interview patients or their family members in the patient's home and discuss the patient's health, lifestyle and drug therapies. It is intended for the pharmacist to observe the patient's management of their medications and to educate the patient so that the quality use of medicines can be achieved. The pharmacist reviews the patient's drug therapies for drug interactions, adverse effects and inconsistencies with the current published views on therapies. Home medicines review can:

- clarify and assure patients about proper use of their medications.

- increase the patients understanding of their physicians clinical intentions. 
- promote co-operation between the patient and his clinician.

- promote better patient compliance with medication regimens and dosages.

- maximize health outcomes from treatment provided.

A patient may have a home medicines review if that patient is living in their own home or in private monitored accommodation. Criteria for a patient to have a home medicines review are one or more of the following:

- Currently taking 5 or more medications.

- Taking more than 12 doses of medications daily.

- Significant changes made to medication regimen in the last 3 months.

- Medications with a narrow therapeutic index or medications requiring monitoring.

- Symptoms suggestive of an adverse reaction.

- Sub-therapeutic response to treatment with medicines.

- Suspected non-adherence to prescribed medications or inability to manage medication related therapeutic devices.

- Having difficulty managing their own medicines.

- Attending a number of different doctors, both general practitioners and specialists.

- Discharge from a facility or hospital in the last four weeks.

- Other medication issues or problems.

\section{Documentation}

Pharmacists are obliged to ensure safe and effective use of drugs to maximize patient outcomes. Documentation conveys information for use in patient care and it serves as a tool for communication among health care professionals. Documentation in pharmacy records may provide a degree of risk reduction, used for research and for education purposes. Pharmacy services provided to patients should be documented in the patient medical record. Pharmacists, as an integral member of the health care team, should document the care provided to patients. Such documentation is vital to a patient's continuity of care and demonstrates both the value of the pharmacist's services and accountability of the pharmacist [2]. Moreover, documentation may increase the likelihood of reimbursement and may also be used in legal proceedings as evidence. Clinical recommendations made by a pharmacist on behalf of the patient, as well as actions taken in accordance with these recommendations, should be documented in a permanent manner that makes the information available to all the health care professionals caring for the patient. Pharmacists are authorized to add notations in patient medication records for the purpose of documenting their findings, assessments, conclusions, and recommendations. Significant clinical recommendations and related actions should be documented in the appropriate section of patient records in accordance with department policy and guidelines. An outpatient pharmacy staff may need to document the following patient relevant information:

1. A summary of the patient's medication history as seen in medication reconciliation form including medication allergies and their manifestations.

2. Consultations provided regarding the patient's drug therapy selection and management.
3. Actual and potential drug-drug, drug-food, drug-laboratory test, and drug-disease interactions.

4. Physicians' new or oral changes of orders received directly by the pharmacist.

5. Adjustments made to drug dosage, dosage frequency, dosage form, or route.

6. Drug related problems that warrant surveillance and extra care.

7. Drug monitoring findings, including recommendations of therapeutic appropriateness of patient's drug regimen and method of administration.

8. Modifications done to eliminate therapeutic duplication in the patient's drug regimen.

9. Degree of patient compliance with the prescribed drug regimen.

10. Clinical and pharmacokinetic laboratory data pertinent to the drug regimen.

11. Actual and potential drug toxicity and adverse effects.

12. Provided patient and family education and counseling.

Pharmacists are required to meet established criteria for legibility, clarity, lack of judgmental language, completeness and appropriate use of pharmacy standard formats throughout documentation process in order to fulfill the purpose of communication and provision of needed information [3]. Pharmacists are responsible to ensure that patient privacy and confidentiality are safeguarded and the communication is concise and accurate. Nonjudgmental language should be used, with care taken to avoid words that imply blame (e.g., error, mistake, misadventure, and inadvertent) or substandard care (e.g., bad, defective, inadequate, inappropriate, incorrect, insufficient, poor, problem, and unsatisfactory).

\section{Challenges and Implications}

Patient satisfaction is a worthwhile goal of health care service and it creates a lot of reasons for implementing research, plans and innovations to achieve it for a better quality of life [4,5]. There has been an increasing focus on the evolution of the role of the pharmacists from traditional drug dispensing to a more active and participative role in safety, risk management, and other medication related consultation activities [6,7]. Pharmacy should always be one the move to improve quality of provided services including safety, timeliness and effectiveness in a caring and respectful environment. Patient-focused activities are provided from admission to discharge and continue on an outpatient basis through ambulatory care services. Barriers to optimize pharmaceutical care in an outpatient pharmacy are illustrated in the following diagram (Table1).

Ambulatory care pharmacies are required to provide pharmacist consultation to patients with new or refill prescriptions. It has been shown that the overall satisfaction of patients with pharmacy services is closely related to their satisfaction with waiting time [8]. Attempts are made to reduce the time focusing in increasing staff members, increasing in the number of payment units or modifying pharmacy setting. All these attempts so far have either a limited success in improving the issue or shortening waiting time and compromising other components such as counseling, education and medication reconciliation. Previous studies have found generally favorable evidence for pharmacist consultation services on various outcomes such as patient medication adherence $[9,10]$ reduction in hospital admission, mortality, overall 


\begin{tabular}{|l|l|}
\hline \multicolumn{1}{|c|}{ Contextual level } & \multicolumn{1}{c|}{ Individual-level } \\
\hline Physical environment & Sociodemographics \\
\hline Social environment & Phychosocial \\
\hline Social environment & Adherence \\
\hline Organizational & Beliefs, Attitudes \\
\hline Economic resources & Income \\
\hline Societal \& Political & Age, Personality \\
\hline
\end{tabular}

Table 1: Barriers to optimize pharmaceutical care.

health care costs $[11,12]$ as well as clinical benefits particular in chronic conditions $[13,14]$.

\section{The Bright Side of a Nagging Issue}

This issue denotes that the institution has customers and it is watched out and it is doing an important job to the community. Though the majority of ambulatory pharmacy customers are dissatisfied with the long waiting time before their prescription medications are prepared and received with proper counseling, it is still good to have customers to run the business. This also means that the institution is important to the community and it is a focus of concern which means that the public will appreciate efforts made to improve service. Within this context, there is a tremendous opportunity for growth and an inviting environment to try new ideas and introduce additional expansions.

\section{The Negative Side}

Community patience will not remain forever. This is considered as an alarm to business success since long waiting time has been given as a reason why some patients do not have their prescriptions filled in a particular pharmacy [15]. Patient pharmacy mean waiting time has been defined as 'the length of time from when the patient entered the pharmacy to the time the patient actually received his or her prescription and left the pharmacy [16]. Queues start to form when the rate of patient arrival at the pharmacy is greater than the service provision rate. A patient's experience of waiting can radically influence his/her perceptions of service quality. A considerable part of the patient waiting time in the hospital can be accounted for by delay of pharmacy dispensing procedure until patient in having their prescriptions sheets filled. This fact shows the significance of waiting time on pharmacy reputation and leads to a loss of opportunities to make profits. Excessive patient waiting time undermines pharmacy efficiency of provided services and patronage. Such delay leads to patient dissatisfaction and thus may eventually result in loss of patronage in a competitive health care environment. It can also lead to poor patient compliance with insufficient instructions given at the pharmacy. Substandard outpatient pharmacy performance may distort organization's image, be a cause for continued blame and leads to interdepartmental uneasiness. Within the department of pharmacy, delay in serving patients cause staff conflicts or may give strong reason to staff to look for a quieter and less physically exhausting jobs or locations. Consequences are deteriorating and frustrating to both clients and employees. The negative effects of stress on pharmacy staff due to unfulfilled demands may lead to poor decision making, deteriorating interpersonal relationships, reduced creativity and individual apathy. Moreover, delay does not support utilization management and has negative impact on facility services including floors, bathrooms, telephones, IT systems, security and parking. Crowding represents a potential for medication errors and incomplete service leads to patient non compliance.

\section{Reengineering}

"Reengineering is the fundamental rethinking and radical redesign of business processes to achieve dramatic improvements in critical, contemporary measures of performance such as cost, quality, service and speed" [17]. Increasing staff and dispensing windows or extending service hours may only and partially solve the problem of long waiting time. It does not fit completely with proper counseling and keeping customers' privacy. What is actually and community expected is a system to reduce the probable causes and finds possible solutions to increased waiting time for patients and prescription errors. There is a consistent need for a breakthrough action to address the core issue and achieve positive outcome in all areas. A whole reformation of the dispensing process is required through the creation of an effective model. Reengineering is mandated in this situation. Healthcare reengineering is a powerful courageous methodology that helps organizations reorder priorities, provide more cost-effective care, and increase the value to customers' money and time. It is driven by what the customer wants and what the market needs. System wide reengineering integrates three levels of activity:

1. Managing community and health plan partnerships.

2. Consolidating overlapping delivery system functions among participating providers and vendors.

3. Redesigning administrative functions, clinical services, and care giving programs to improve health status.

Reengineering is a critical core competency and requisite skill for health care organizations if they are to succeed under managed care in the future. The 8-steps system is a schematic path to manage outpatient pharmacy that is optimized to yield the most possible outcome in:

1. Reducing patient waiting time.

2. Effective counseling.

3. Maintaining customer's privacy.

4. Improving compliance.

5. Effective medication reconciliation.

6. Reducing medication errors.

7. Strengthening pharmacy - customer rapport.

8. Reduction in wastes and redundancies.

9. Increasing staff expertise and knowledge.

10. Turning outpatient pharmacy into a medication clinic.

The traditional system of a pharmacist standing behind a counter or widow engraved in walls is no longer efficient to meet modern requirements to optimize pharmacotherapy of patients. It still represents a hurdle between customers and pharmacy staff. Patients cannot be assured that they leave pharmacy with their prescribed medications fully aware and ready to comply for the treatment. An innovation is needed to meet standards and improve the quality of pharmacy service. The old name of outpatient or ambulatory pharmacy should be replaced by a candidate name for the next era into Medication Clinic. The service place should be turned into a real live encounter between service provider and the client (Chart 1).

\section{The 8-Steps System}

The system is mainly subjected to activities and encounters that take place between pharmacy staff and customers away from pharmacy interior area. Outpatient pharmacy interior layout definitely has its effect on the flow of work and parallel efforts should be done for reformation if necessary. The innovation in the dispensing system 


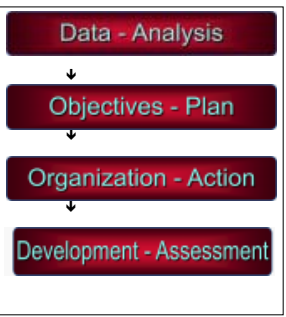

Chart 1:

majorly takes place outside the pharmacy adjacent area. The project of reformation comprises eight basic steps in order to achieve the anticipated outcome from an operating outpatient pharmacy. The eight steps are briefed as:

1. Have relevant data.

2. Analyze.

3. Manage your objectives.

4. Manipulate your plan.

5. Organize.

6. Undertake action.

7. Develop.

8. Assess.

Each step of the project has its own confinement and is connected to be smoothly transitioned and overlapped to the next step in order to consolidate project integration. Adaptation to each organization or healthcare facility is an inevitable necessity to the success of the project provided that the main concept and objectives are intact and aliened with organization's mission and vision. A detailed description of each project eight steps is illustrated below.

\section{Have relevant data}

For an identified issue to be addressed, related data have to be collected at the first step. Complaints, conflicts and demands have to be compiled and considered in mind to come with an actual size and effects of the issue in concern. Patients may be less able to judge the technical quality of the care they receive, but they do judge their social interaction with the pharmacist. A delay is more likely to come up at the end of a process component due to a break in the workflow. Decrease in the number of process components of the dispensing procedure should lead to decrease in delay and subsequent decrease in total waiting time. Overall, patient satisfaction has been found to be more closely related to satisfaction with waiting time and whether the pharmacy staffs are helpful and caring [18]. Pharmacy professionals must increase patients' awareness of the value of pharmaceutical care services and make it important to their judgment of satisfaction [19]. Occurrence reports, customer surveys, anecdotal patient encounters, related staff numbers, pharmacy layout, prescription numbers, daily senses, competitors, complaints, frontline staff concerns and financial statements are examples of necessary data to look carefully at in order to shape a defined issue that needs to be improved and to determine why a breakthrough is needed to achieve the desirable outcome.

\section{Analyse}

Data are primarily collected and harmoniously grouped for analysis using quality improvement tools to illustrate actual facts and solid information on the specific issue to come out with a defined decision. Data may include staffing deployment and skills, number of shifts, prescriptions average rate, operational costs, area population, and past surveys. Organizational chart and process flow chart are used to assess work flow and have a general scope on the ongoing process. Needs assessment is done to establish a strong reason for pursuing into the coming change.

\section{Manage your objectives}

Pharmacy leadership determines the short and long term departmental goals, which are consistent with and focus on fulfillment of the department's and institution's mission and vision. Objectives have to be clear and well defined in order to begin from an established base to go forward and gain organization leadership commitment and support. One always should ask the question; why there is a concern? And more importantly; how far this may impact the level of service?

\section{Manipulate your plan}

The plan is generated to achieve stated objectives in a suitable time frame using affordable resources and manpower. The main three elements of the plan are (a) design, (b) staffing, and (c) process.

Design: "First we change our structures and afterwards they shape us" Winston Churchill. The core idea of the project proposal comes from innovation in design of the outpatient pharmacy and dispensing process performed by properly oriented trained staff in order to yield the prospectus outcomes. To evaluate the structural characteristics, one would try to decide if the physical aspects of the healthcare facility and the characteristics of the personnel are appropriate for these particular services provided to clients. Design should be adapted to each facility to fit with the service characteristics and customer needs and expectations "Think global and act local". Design effectiveness is evaluated by functionality, impact and capability to meet standards and open venues for continued improvement. Design includes space planning, lighting, noise control, privacy issues and safety. Design includes signage, furnishings, and selections of color and texture. The color scheme should be appropriate to the patient, pharmacists, other staff and the community. The design must relate and help define the term "high-quality care". The design, therefore, must balance technological needs and human needs. Healthcare solutions must be tailored to local circumstances expressing the local regional elements to the design, materials, colors, textures and patterns, as this is a good start towards patient and staff comfort and satisfaction with the environment. The Pharmacy should be located near the main entrance for easy access. It should not be near high volume queuing areas such as laboratories and radiology. Accessibility to pharmacy area should be easy without any hurdles or unsafe obstacles.

Avedis Donabedian's structure/process/outcome (SPO) model is frequently cited in research on measures of healthcare quality. Donabedian defines structural measures of quality as the professional and organizational resources associated with the provision of care, such as staff credentials and facility operating capacities [20]. The relationship between structure, process and outcome is causal i.e. good structure should promote good process and good process in turn should promote good outcome.

\section{Structure of Care $\rightarrow \quad$ Process $\rightarrow \quad$ Patient Outcome}

Access of care is a paradigm that should precede "structure of care" to improve quality of provided care and long patient waiting times in an outpatient pharmacy represents a clear example. Department performance is influenced by supporting structural factors and design 
to enable staff doing required jobs in an effective way. In a study with physicians, the importance of the level of education on perception of services was shown by the fact that patients who had higher education assessed the work of physicians more critically [21]. This fact can be applied to all healthcare services including pharmacy. A design is needed to allow closer relationship between pharmacists and clients with preservation of privacy and confidentiality. Local traditions and customs have to be considered in mind when setting a design as a show of care and respect to patients. A design for the project occurs when the complete project team formed by key executive personnel that may be including high ranked staff from Pharmacy department, Customer service, Quality and innovation, Patients relations, Purchasing, Engineering, and Finance. An executive team is required to finalize the initial framework and plan input in the early schematic phase. The team is required to work together from the inception of the project and must ensure that all engineering disciplines are coordinated with the architectural and process requirements of the project. Care is taken to provide design solutions that meet the construction standards, space requirements, equipment layouts, and vertical and horizontal clearances required by the institution. All conflicts between engineering systems and safety requirements should be resolved by the final design development submission. The drawings and specifications shall be complete, clear, and coordinated. Pharmacy design must reconcile the need for security with the functional need to dispense medications throughout operating time. Suitable illustration of the area sections is needed including relationship to adjacent surroundings. In developing the preliminary design, a special attention is taken to conditions where a new structure joins an existing structure. Setting should afford:

1. An environment to promote department's mission statement.

2. Pleasant accessible place to work, serve, and represent a satisfactory client encounter.

3. Effective layout to maximize staff performance and handle patients physical, psychological, and emotional needs in the best possible way.

4. Satisfying encounter and experience though provision of pharmaceutical care including drug therapy monitoring if applicable, counseling, patient and family education and medication reconciliation.

5. Respect and care for local customs and traditions. Segregation may be done through establishing two separate identical areas for males and female patients in certain urban and conservative communities.

6. Reduction in aggression behaviours towards staff.

A core challenge in the proposed design is to develop a workplace that is flexible, appropriate and affordable in the short, medium and long term in the context of the current public demand challenges. The design should be flexible enough to provide services into the future without the need for major internal modifications.

Design specifications: a) Windows and counters: The system is based on the conception of providing service in close friendly encounters. The traditional bank teller design is no longer supportive to the desired level of care. Windows may still be kept within the design just to serve customers who are not been received in pharmacy reception area. Dimensions should be as wide as possible for better communication or even replaced by reception counters. Windows quick encounter that may only as a delivery of patients prescriptions should be followed by table encounter if related to prescription filling.
Segregation between males and females may be considered according to local traditions and customs.

b) Waiting area: This area is where patients wait for their prescriptions for filling. There will be a central entrance for all clients of the pharmacy, giving access to waiting area and windows or counters. The perimeter includes the service windows or reception counter and pass-through compartments or the especially designated stations used in the open concept. This area is contiguous to the prescription receiving area. The functions performed here include: welcoming customers, receiving the prescription and directing customers to their tables where they are going to receive their medications along with the rest of necessary services.

c) Doors: A door or two should be present and directly opened to the welcome area to facilitate movement and shorten distance for pharmacy staff to access the welcoming area.

d) Fence: Pharmacy welcoming area should serve as a kind of lobby, reception, saloon, café or an environment that gives the impression of a place to conduct a pleasant and fruitful meeting with cherished customers. The welcome area may be defined using suitable paravan, septum, mesh or even ropes and stands, just to confine the area and maintain privacy.

e) Tables: Numbered tables with two or three identical chairs are put in the waiting area to meet and counsel customers. Tables may be provided with edge septum to ensure privacy. Stationary should be kept in each table to help educating patients and fill documentation forms. Tables are the place for confidential consultation between the pharmacist, patient and family or friends. The need and purpose of the patient's medication will be reviewed and the regimen clarified.

f) Middle counter: A long counter is set in the center of the waiting area to serve for keeping literatures, magazines, decorations or welcome refreshments. This counter may be eliminated in case of a facility with low work volume or limited space.

g) Trays and boxes: Transparent polyvinyl trays are used to individually prepare prescriptions inside pharmacy. After final checking, drugs are transferred into bags or foam boxes and carried by the counseling pharmacist to the designated patient waiting in the specified table with the same number being for all; table, tray, bag or foam box.

h) ID cards: Staff ID cards should be attractive and clearly visible.

i) Patient number card: A laminated card carrying customer's number is handled as soon as prescription is received and the patient or his companions are directed to the waiting table with the same number. The customer should be informed on what to expect regarding waiting time or things related to billing or reimbursement.

j) Video display screens: TV screens may be installed to entertain waiting customers and can be used to display general health educational materials.

Evidence based design: Evidence-based design may be defined as the process of basing decisions about the built environment on credible research to achieve the best possible outcomes [22]. Customer satisfaction is a major concern for healthcare providers. In general, customer satisfaction is multifactorial and is considered a part of overall consumer behavior which evolves over time and is influenced by many factors. Several key factors that greatly influence satisfaction include consumer's expectations, attitudes, and intention about the service provided. After implementation of the 8-steps system, a follow 
up study is to be carried out to further evaluate the difference in areas of outcomes and demonstrate evidence of identified significance. The 8 -steps system is proposed to improve outcomes in the following areas:

1. Courteousness: Friendly and courteous encounter with clients through "meet, greet and treat". This is to "confront at the front "not just standing behind walls and windows.

2. Waiting time: As an important determinant of patient satisfaction, waiting time has gained increasing attention in the field of health care services. Customer satisfaction has been defined as the difference between the customer's perceptions of the experience and his or her expectations, which is many times based on past experience. Through a better design and seamless flow of work, it is expected that patient mean waiting time will be drastically reduced.

1. Communication: Efficient ergonomics can influence the ability to use information and perform tasks. Windows and counters may affect the degree of customer's discomfort. Visibility and physical disorders has been shown to impact dispensing errors when items become difficult to differentiate and patients are in a hurry to end up their long standing to finally have their own prescriptions filled. Space design can also influence lighting, noise, and interruptions. Environmental barriers such as septum, glasses, and noise may bring a potential for misunderstanding and lead to medication errors. Human barriers such as hearing impairment, vision, language, psychical disability and personal uneasiness need to be addressed at each pharmacy encounters with patients.

2. Rapport: Closer distance between pharmacists and patients is maintained and this would expectedly result in better rapport and gained trust. A friendly close encounter is achieved to avoid physical and human barriers.

3. Counseling: It is safe to say that the traditional methods employed by pharmacies to improve customers satisfaction (e.g., comfortable waiting area, coffee, magazines and video display screens) would be of limited benefit in pharmacies that fill in excess of 400 prescriptions per day and have patient waiting times that commonly exceed 1 to 2 hours. Areas of counseling, reconciliation and education are severely compromised. The 8 -steps system is innovated to address all issues related to ambulatory pharmaceutical care in one situation.

4. Adherence: Patients satisfied with their pharmacy services are substantially more likely to adhere to their medication [23]. Patients who have improved their healthcare outcomes are more likely to evaluate their healthcare providers, (e.g. physicians, nurses and pharmacists) more favorably.

5. Credibility: The new design releases a message of the preoccupation of management with customer's needs and expectations. It also represents a practical way to gain credibility through direct and genuine work to achieve organization consistency with declared mottos such as "we care".

6. Utilization management: The 8-steps system is expected to ease and accelerate pharmacy operations through maximization of staff effectiveness. Pharmacies that experience high-volume prescription workload frequently have difficulty in managing workflow and waiting times because of the old "bank teller" design. Reducing customers waiting time into half period can lead to a substantial improvement of chronic parking availability problems.

Staffing: Outpatient services are one part of a comprehensive pharmacy services. While the function of medication supply is fundamentally the same in all pharmacy sectors, it is important for pharmacists working in an outpatient setting to understand the clinical role in this section. Staffing requirements are determined by expected daily patterns of physician order generation and refill rates. Sudden changes in staffing requirements may be accommodated by:

\section{Overtime.}

2. Temporary hiring of supplemental staff.

3. Shifting staff and work among work areas.

Outpatient pharmacy performance should be tracked bi-weekly and discussed monthly in combination with customer service, quality and innovation, human resource, and financial data. Direct patient care or pharmaceutical care is provided by all employees of the department of pharmacy services. The team that provides services in outpatient pharmacy may include:

1. Director of pharmacy services.

2. Clinical pharmacy manager.

3. Pharmacy operations manager and process coordinator.

4. Clinical pharmacists and clinical pharmacy specialists.

5. Staff pharmacists.

6. Pharmacy technicians.

7. Pharmacy residents and students.

8. Inventory control specialists.

9. Administrative assistants.

10. Clerks.

11. Technology support personnel.

12. School of pharmacy faculty members.

Pharmacy clinical role may include patient and family education, medication reconciliation, drug monitoring and optimizing pharmacotherapy. Evidence suggests that having clinical pharmacists manage medications can improve patient care and, in some cases, lower costs [24]. BSc. Pharmacists with more than five years experience can be trained and educated to effectively perform medication management with minimal supervision. Ideally, clinical pharmacists, specialty board certified pharmacists and senior pharmacists should be assigned to perform the final phase (patient encounter) during prescription dispensing process. They are specially expected to afford outpatient medication management to patients with tertiary care and chronic illnesses such as metabolic syndrome, neurology and psychiatry, those on anticoagulants and oncology patients. This practice has been proven to lead to improved outcomes and lower costs. Motivating staff may take place through conducting training program for staff pharmacists ending up with a credential of "pharmacy counselor" to improve service and motivate pharmacy staff to work in pharmacy clinic with certain privileges. A ratio of 3 staff to 50 customer (adjusted to case mix index) per 8 hours shift is expected to yield a satisfactory work flow and satisfactory outcomes. Managers, clinical pharmacists, staff pharmacists, technicians and assistants are all needed to commit to the success and sustainability of the 8-steps system. Gaining buy-in 
from those doing the work is a key to meaningful improvement and sustainability. Organization and pharmacy department leaders should show appreciation and honor the work of the front line staff.

Process: Process includes the operational types of services and procedures available at pharmacy clinic. The structure-processoutcome paradigm is presented as a framework for quality assessment of pharmacy clinic. Structure should be assessed at periodic intervals because it identifies the potential for the provision of quality care. Process, the care that pharmacists provide, must be documented and linked to outcomes before either structure or process can be used to make inferences about the quality of pharmacy clinic. Technical and interpersonal processes should be examined periodically. Outcomes require an interdisciplinary approach that not only considers other medical care inputs but also recognizes the psychological, economic, and social factors that affect health status and quality of life of pharmacy customers. Process and outcome must both be assessed to distinguish the contribution effect of pharmacists from that of other healthcare providers. All staff of the ambulatory pharmacy should participate within their defined roles in the application of the new system and engage proactively in the success of the process.

Leadership involvement, commitment and leading by example is a crucial factor for the success of the 8-steps system. Chairman of pharmacy and pharmacy senior staff are the first personnel to lead the process. Beside the allocated staff, Head of pharmacy, Section Heads, Managers, Clinical pharmacists and any active pharmacists in various pharmacy sections may be assigned to counsel and dispense a daily number of prescriptions ranging from only one to several prescriptions per shift in order to share expertise and promote partnership within the department. A register is generated to document prescriptions dispensed by non permanent staff on a daily basis for consideration on annual appraisals. The procedure steps can be broadly implemented in order sequenced as follows:

1. Welcoming: Customers are welcomed as in front as it can be. A standing staff may be ready to meet and treat. Customers are informed what to expect. A table number card is given to the customer and the same number table is shown to the customer and he/she is told that after preparing prescription or bill, a pharmacy staff is going to come out to continue service either by handling a bill to be paid at cashiers office or directly dispense medications according to insurance card type.

2. Transaction entry: Pharmacy staff performs entry in billing system.

3. Payment: Patients or their companions pay the bill at cashiers desk and returns to pharmacy with the receipt.

4. Preparation and checking: A foam box carrying institution logo is used to keep medications and further kept inside an environment friendly bag. Patient table number is fixed and package is put in line ready for pharmacists to take and go out to meet customers and continue proceeding to next step. Following profile review, pharmacists should highlight, make a note, or use an auxiliary label on the prescription bag or in the computer claims system as a reminder that some aspect of the drug regimen has changed. New prescriptions for devices such as inhalers and injectables for counseling opportunities should be flagged.

5. Getting out: Customers are re-identified. Clients are identified as new or frequently receive care. As a safety precaution, pharmacists should use of at least two unique identifiers for each new prescription order and upon consultation when patient is unknown to them to ensure that patient data is correct and correct patient receives correct medication. Alternatively, verification of patient name in addition to at least one other identifier, such as address, date of birth, or phone number when patient is unknown to pharmacist can be done as well. Clients may be asked open ended questions on how they feel about being served and how is their day within hospital premises. Story of medication is about number of items, why they are prescribed and what is expected as an outcome. Patients should be informed about any manufacturer changes or changes to the medication regimen. Each prescription bottle is opened to confirm that the medication looks the same to the patient.

6. Counseling and education: A positive correlation exists between pharmacist time required for counseling patients and the characteristics of the services provided. Guided by a documentation form. Counseling may include :

7. Dosage and route of administration.

8. Duration of therapy.

9. Proper storage

10. Refill information

11. Drug potential interactions with concurrent drugs, food, drinks, herbals, smoking, disease, laboratory and physiology changes.

12. Past allergies.

13. Monitoring and managing of adverse and side effect.

14. Modification in life style to maximize medications effects.

15. Therapeutic duplications.

16. Expected outcomes.

17. Untreated conditions.

18. What to do in cases of missed doses or mistaken overdoses.

19. Over/under-dosing, taking renal and hepatic function into account.

20. Care for special age populations.

21. The importance of compliance and adherence to regimen.

22. Demonstration on devices and manipulations if needed.

23. Instructions are reaffirmed are restated back.

24. Drug related financial and social issues.

25. Medication reconciliation: Medication reconciliation is the process of comparing a patient's new medication orders to all of the medications and complementary products that the patient has been taking. This reconciliation is done to avoid medication errors such as omissions, duplications, dosing errors, or drug interactions. It should be done at every transition of care in which new medications are ordered or existing orders are rewritten. Transitions in care include changes in setting, service, practitioner or level of care. This process comprises five steps:

1. Developing a list of patient current medications. 
2. Developing a list of medications to be prescribed.

3. Comparing the medications on the two lists.

4. Making clinical decisions based on the comparison.

5. Communicating the new list to appropriate caregivers and to the patient and family after each visit or discharge (Form A).

Accurate, complete and ongoing medication reconciliation can prevent numerous potential prescribing and administration errors. Failure to reconcile medications may occur by the practice of writing orders, such as "resume pre-op medications", which are highly error prone and are known to result in adverse drug events. Medication errors related to medication reconciliation typically occur at the "interfaces of care" when a patient is admitted, transferred, or discharged from a health care facility.

6. Documentation: Consistent profile review of all patients (to include documentation of weight and dose/weight or dose/ body surface area for pediatric patients) should be done at each encounter in order to update information, optimize pharmacotherapy, and to identify drug-related problems and standardize pediatric dose-checking. Documentation of all activities and actions taken should be readily retrievable and maintained in the pharmacy for 5 years or according to guidelines.

7. Bye: At the end of the encounter, the next pharmacy or clinic visit and what to expect should be notified. A business card is handled to maintain communication if needed.

Organize: Organizing the project and putting it into effect needs the orchestration of an interdisciplinary team and plotting a time chart for execution. Project organizing is the art of directing and coordinating human and material resources throughout the life of a project to achieve predetermined objectives of scope, cost, time, quality and participation satisfaction. Functions of organizing project may include the following:

1. Matching of project objectives and plan including delineation of scope, budgeting, scheduling, setting performance requirements, and selecting project participants.

2. Maximization of efficient resource utilization through procurement of labor, materials and equipment allocation according to the prescribed schedule and plan.

3. Coordination and control of planning, design, estimating, contracting and construction in the entire process.

4. Development of effective communications and mechanisms for resolving conflicts among the various participants.

Undertake action: This step includes (a) Pharmacy restructure, (b) Grouping up of materials and equipment, (c) Training of potential staff followed by (d) A pilot rehearsal to fit with each organization and finally (e) Implementing the project in a suitable date.

a. Pharmacy restructures: An adapted designed layout is to be carried out to shape the whole outpatient pharmacy in order to yield outcomes that are expected to achieve and promote organization mission and vision.

b. Equipment and materials: Except for redesigning the outpatient pharmacy adjacent area for meeting customers which is mainly done by the engineering department, a list of materials should be generated and procured through liaison with the purchasing department to be ready at time of implementing the project.

c. Training: Primarily, leaders and supervisors are introduced to the system and staff is trained to manage expected scenarios, filling forms, required activities of medication management and documentation. All pharmacists are trained to provide patient education, participate in Adverse Drug Reaction (ADR) and Medication Error program, participate in investigational drug research, drug and therapeutic policy development and implementation, and other cost and quality enhancement programs and projects. Pharmacists' interventions are to be recorded and documented related records. Clinical pharmacy specialists and clinical pharmacists provide direct patient care throughout the institution and their level of education and expertise is particularly needed in ambulatory settings. These practitioners are active integral members of medical service teams and assist the team by providing services including:

1. Collaboration with physicians and other health care providers.

2. Optimizing pharmacotherapy outcomes through reviewing patient medical record, drug selection, resolution or prevention of drug-related problems.

3. Pharmacokinetic assessment and drug dosing.

4. Drug information.

5. Provision of proficient and compassionate direct patient care through patient interviewing, medication counseling and education.

6. Preparation of sterile admixtures

7. Management of drug and healthcare related research studies.

8. Educating other health care providers and students.

d. Pilot application: The system can be implemented on a small scale and then expanded to all hospital outpatient pharmacy outlets. Weekends or other low work load timings may represent good periods to start system application.

e. Implementation: Shortly, one or two weeks after pilot application, the project should be implemented fully on the designated facility. Project implementation phase is entered once the groundwork is complete within the plan main framework. Regular meetings are usually required in order to clarify and facilitate work flow.

Develop: Being a newly introduced project, development and improvement should take place to encounter obstacles and facilitate change. Pre and post surveys may be used to compare outcomes and help find decisions. Improvements can be made in all phases of the system and they should be adapted to each facility. Usually, the leaders of the department of pharmacy foster the process of development through the application of behavior-oriented interdisciplinary theories aligned with strategic plans. Always there is a room for further innovative ideas to enrich the ever needed improvements.

Assess: After a considerable period probably three months from implementation, an assessment should be made to evaluate the project outcome by comparing pre and current results. Comparing base conditions with projected conditions through benchmarking or auditing should be carried out using reliable procedures for collecting data in order to get correct results and consequently come out with promising decisions. A run chart may be used to plot patients waiting times and outliers are identified and analyzed for further improvement. Findings should be reported to senior management and relevant 
information should be available to all stakeholders and decision makers. Service can be extended and redeveloped according to degree of success and resources availability. Let facts speak loudly.

\section{Expected Outcomes}

Though structure and process are easier to measure; outcome is more important. Outcome measures are the desired states resulting from care processes, which may include reduction in morbidity and mortality, and improvement in the quality of life. There are two types of interdependent outcomes; technical and interpersonal. Technical outcomes encompass the physical and functional aspects of care such as the absence of postsurgical complications and the successful management of hypertension and other chronic illnesses. Interpersonal outcomes encompass dimensions of the art of treating patients such as satisfaction with care and the influence of care on their quality of life as perceived by the patient. The 8 -steps system has the potential to influence organization wide image and reputation through the following expected outcomes:

\section{Organization}

Success in one hospital department will certainly affects other departments by conduction and the net result would be a wholesome of improvement in performance organization wide. The proposed system has the potential to enhance new models of care though the expansion of health promotion programs and enables absorption of increases in patient list size and future expansion. The innovation may stimulate civic pride, enhance the locality, and provide a welcoming environment that promotes well being. There is a chance for the system to enhance the relationship with other partner organizations to deliver health services through mutual collaborations.

\section{Customers}

Impressive results in reducing patient mean waiting time are expected. Patients will have the impression that staff are kept busy to serve then as soon and as effective as possible. The read message is that pharmacy provides the best quality care pathway, improves patient experience, and brings care closer to home. The proposed system should improve service and patient experience as being located in a convenient and accessible location. The development of a newly designed ambulatory pharmacy will ensure long term sustainability of the health system, particularly in relation to issues such as: population growth, an increasing ageing population, ability to implement changes in evidence based practice, capacity to adapt to advances in clinical technology and medicines, and providing opportunities for training and hence a sustainable workforce to support future health reforms. The proposed project represents an excellent opportunity for the delivery of integrated healthcare where networks of providers can come together to provide a greater and more sustainable resource of health services for the local community.

\section{Pharmacy staff}

It is identified that the substantial workload within the outpatient pharmacy is contributing to unacceptable stress levels and lack of job satisfaction for staff, and less than optimal patient care. The 8-steps system is expected to be of good relief of stress and add excitement and sense of entertainment in working area. The system is expected to expand the domain and diversity of staff expertise. The new system encourages feedback from employees and activities that could be done to improve the system. The system ensures that every employee believes in the quality improvement and becomes a part of the system. The new system will provide a much better working environment for pharmacy staff and will improve the practice's ability to attract new talent to grow the workforce. The new system maximizes workforce contribution and should lead to reducing health inequalities and promote healthier communities by integrating care and promote partnership between healthcare professionals. It is also supposed to ensure long term sustainability of the health system, and providing opportunities for training and hence a sustainable workforce to support future health reforms. The new system design has the capacity to maximize staff productivity and illustrating their competencies and enthusiasm through change in pace and avoidance of stagnating, boring and routine style performance.

\section{Patients counseling}

Since medications represent an integral part of disease management, medication therapies and their delivery systems are becoming more complex, there is a greater potential harm to the public and a greater need for patients' medication use to be managed by qualified pharmacists within agreed standards of care in a supporting environment. In the 8-steps system, Pharmacists are expected to provide direct care to high-risk patients, including diabetics, those on anticoagulants, psychiatric patients, oncology patients and others. Most patients are indigent or elderly and pharmacists should be able to perform medication reconciliation, monitor patients according to key indicators, adjust medication doses as necessary, and educate patients and providers about managing these conditions. The system provides a certain opportunity for conducting and updating "Drug Regimen Review" process at each patient-pharmacist encounter. This proposed system will provide a safe, clean and comfortable fit for purpose environment which will ensure that the patient is treated with privacy and dignity which in turn will contribute positively to the wellbeing of patients, staff morale, and the efficiency of service delivery. The 8-steps system is expected to lead to significant improvements in health outcomes, including improvements in blood glucose levels among diabetics and international normalized ratio levels among those on anticoagulants, prevention of many adverse drug events, and meaningful cost savings due to reductions in emergency department visits and inpatient admissions. The proposed system represents a promising setting to alien with strategic priorities for national action as identified in "Healthy Future" projects such as:

1. Increasing participation rates in physical activity.

2. Reducing unhealthy eating.

3. Stopping the growth in harm from alcohol and drugs.

4. Reducing accident and injury rates in domestic and traffic.

5. Improving mental wellbeing.

6. Improving health at work place.

7. Increasing vaccination rates to recommended levels of immunization.

8. Promoting smoking cessation.

\section{Medication errors}

Asking patients about allergies and adverse drug reactions at each visit and documenting changes or updated information should have a great effect in avoiding possible errors. On the other hand spending extra time on patients counseling and education is expected to improve patients understanding about their medication and results in fewer missed doses or over/under use of medications. The new design allows for more chance to give the needed special attention at look-alike, sound-alike medications to be identified and verified. 


\section{Medication reconciliation}

Especially for polypharmacy patients and those with chronic illnesses such as asthma, chronic obstructive pulmonary disease, diabetes, hypertension, hyperlipidemias, congestive heart failure, HIV or AIDS, osteoporosis, and co morbidities.

\section{Rapport}

Better staff-staff and staff - customer rapport is expected to rake place as a closer relationship is established between pharmacy staff and clients. Meanwhile, increasing the rate of referrals and pharmacy interventions and recommendations to physicians will result in continued communication and sharing of patients related information. This will add to the enforcement of the concept of partnership and integration of provided care. The system opens an excellent opportunity to pharmacists to demonstrate their skills and ability to enhance outcomes, and build relationships with the physicians to cultivate trust.

\section{Utilization management}

The system gives low impact on hospital resources through reduction of redundancies and unnecessary visits. The mission is to assist hospital management in identifying ways to improve processes and enhance the effective deployment of resources (people, equipment, facilities, supplies, and systems) in order to improve financial performance, reduce service turnaround time, and improve customer satisfaction.

\section{Medication risk management}

The system allows for an environment of a continuous quality improvement (CQI) program for medication risk management in order to identify and evaluate quality-related events and improve patient safety through standardization of workflow within the pharmacy and incorporating risk management techniques into daily workflow such as:

9. Flagging medications in pharmacy for which special attention is needed e.g. Look-alike, sound-alike medications.

10. Checking medication expiration dates immediately before reaching patients.

11. Allowing for patients back demonstration on the use of their instrumental medications like inhalers and insulin injections.

12. Performing a final check that the name on the label of the medications that are placed into the bag matches the name on the receipts that will be given to the patient.

13. Verification of phoned prescriptions by repeating the written information back to the prescriber.

14. Comparing names of medications on the manufactured bottles with the names of medications on the prescription labels after filling the prescription order.

15. Creating a documentation system for quality-related events so they can be effectively addressed and improvements can be made.

\section{Cost savings}

Reduced Emergency Department visits, hospitalizations, medication errors will definitely result in substantial cost saving in both med and long term span. The new system aims to ensure the long term sustainability of outpatient pharmacy services in the area, and address key issues such as population growth and the needs of a diverse ethnic population. The new design would also provide an opportunity for pharmacy staff to work collaboratively with the community, clinicians, government and other partners to improve the health outcomes of the community. The new system would help to reduce lost resources through improving integration of services, eliminating redundancies, and consolidating the continuity of care for patients. The system is most likely expected to provide the strategic and service led rationale for the relocation and development of a new designed outpatient pharmacy.

\section{Parking}

Hospital parking is widely recognized as being amongst the commonest causes of complaints by patients, and the most frequently acknowledged and dismissed as being too difficult to resolve. Reducing service time within the hospital will allow for more space and help solving parking's issue to some degree.

\section{Equity}

All patients are entitled to equality of access to services, which both respond to their needs and are of an acceptable standard and quality.

\section{Community QOL}

It is proposed that the new system design would be a modern fit for purpose with the flexibility to adapt to future requirements of our rapidly evolving society. It would alleviate the problems and restrictions of the current service provided and achieve capacity to enhance services and work in partnership with other providers of healthcare services. The total all result is perceived as a better way and better quality of life.

\section{Conclusion}

Reformation of ambulatory (outpatient) pharmacy is becoming global demand to cope with the scope of services and care required to be provided to patients. Pharmacy Clinic merges quality concepts with the practice of pharmacy to replace the existing outpatient or ambulatory pharmacy. The "bank teller" design is no longer suitable to afford a quality pharmaceutical care. The 8-steps system has the potential to turn Pharmacy Clinic into a "pharmacy café" where the encounter between clients and pharmacists is a rewarding experience for both parties.

\section{Conflict of Interest}

This project was created and developed with no financial support or associated conflict of interest.

\section{References}

1. The role of the pharmacist in the health care system (1993) Report of a WHO consultative group, New Delhi, India, 13-16 December 1988 and Report of a WHO Meeting, Tokyo, Japan, 31 August-3 September

2. ASHP Guidelines on Documenting Pharmaceutical Care in Patient Medica Records.

3. Lacy CF, Saya FG, Shane RR (1996) Quality of pharmacists' documentations in patients' medical records. Am J Health-Syst Pharm 53: 2171-2175.

4. Shea AM, Curtis LH, Hammill BG, DiMartino LD, Abernethy AP, et al. (2008) Association between the Medicare modernization act of 2003 and patient wait times and travel distance for chemotherapy. JAMA 300: 189-196.

5. Chand S, Moskowitz H, Norris JB, Shade S, Willis DR (2009) Improving patien flow at an outpatient clinic: study of sources of variability and improvement factors. Health Care Manag Sci 12: 325-340.

6. Worley MM, Schommer JC, Brown LM, Hadsall RS, Ranelli PL, et al. (2007) Pharmacists' and patients' role in the pharmacist-patient relationship: are pharmacist and patients reading from the same relationship script? Res Soc Admin Pharm 3: 47-69.

7. Schommer JC, Pedersen CA, Worley MM, Brown LM, Hadsall RS, et al. (2006) 
Citation: Hammouda El, Hammouda SE (2012) Outpatient (Ambulatory) Pharmacy; an Innovation in Dispensing System to Optimize Performance and Meet Standards. Pharmaceut Reg Affairs 1:101. doi:10.4172/2167-7689.1000101

Page 12 of 12

Provision of risk management and risk assessment information: the role of the pharmacist. Res Soc Admin Pharm 2: 458-478.

8. Lin AC (1999) Identification of Factors Leading to Excessive Waiting Times in an Ambulatory Pharmacy. Hosp Pharm 34: 707-712.

9. Bjorkman I, Viberg N, Rydberg L, Lundborg CS (2008) Health promotion a Swedish pharmacies - views of the staff. Pharmacy Practice (Internet).

10. Lee JK, Grace KA, Taylor AJ (2006) Effect of a pharmacy care program on medication adherence and persistence, blood pressure, and low-density lipoprotein cholesterol: a randomized controlled trial. JAMA 296: 2563-2571.

11. McCombs JS, Liu G, Shi J, Feng W, Cody M, et al. (1998) Kaiser Permanente/ USC patient consultation study: change in use and cost of health care services. Am J Health Syst Pharm 55: 2485-2499.

12. Yuan Y, Hay J, McCombs J (2003) Mortality and hospitalization impacts of pharmacy consultation in ambulatory care. Am J Manag Care 9: 101-112.

13. Paulos CP, Nygren CE, Celedon C, Carcamo CA (2005) Impact of pharmaceutical care program in a community pharmacy on patients with dyslipidemia. Ann Pharmacother 39: 939-943.

14. Jaber LA, Halapy H, Fernet M, Tummalapalli S, Diwakaran H (1996) Evaluation of a pharmaceutical model in diabetes management. Ann Pharmacother 30: 238-243.

15. Somani SM, Daniels CE, Jermstad RL (1982) Patient satisfaction with Outpatient Pharmaceutical Services. Am J Hosp Pharmacy 39: 1025-1027.
16. Worley MM, Schommer JC (1999) Pharmacist Patient Relationship: factors influencing quality and Commitment. J Soc Admin Pharmacy 16: 157-173.

17. Hammer M, Champy J (1993) Reengineering the Corporation: A Manifesto for Business Revolution. Harper Collins, London.

18. Johnson KA, Parker JP, McCombs JS, Cody M (1998) The Kaiser Permanente/ USC Patient Consultation Study: Patient satisfaction with pharmaceutical services. University of Southern California. Am J Health Syst Pharm 55: 2621 2629.

19. Ried LD, Wang F, Young H, Awiphan R (1999) Patients' satisfaction and thei perception of the pharmacist. J Am Pharmacists Assoc (Wash) 39: 835-842.

20. Donabedian A (1966) Evaluating the Quality of Medical Care. Milbank Mem Fund Q 44: 166-206.

21. Miseviciene I, Milasauskiene Z (2002) Patient satisfaction with the work of the hospital medical personnel. Medicina (Kaunas) 38: 559-565.

22. The Center for Health Design (2009) Definition of Evidence-Based Design

23. Ning Yan GU, Yunwei GAI, Joel WHAY (2008) The effect of patient satisfaction with pharmacist consultation on medication adherence: an instrumental variable approach. Pharmacy Practice (Internet).

24. Bond CA, Raehl CL, Franke T (1999) Clinical pharmacy services, pharmacis staffing, and drug costs in United States hospitals. Pharmacotherapy 19: 1354 1362. 